\title{
First report of Sarocladium oryzae causing sheath rot of Oryza rufipogon in Zhanjiang, China
}

\author{
Huang Yongxiang ${ }^{1} \cdot$ Liu Yuelian $^{1}\left[\right.$ Xie Zhihao $^{1}$ \\ Received: 20 December 2021 / Accepted: 22 February 2022 / Published online: 7 March 2022 \\ (c) The Author(s) under exclusive licence to Società Italiana di Patologia Vegetale (S.I.Pa.V.) 2022
}

Keywords Oryza rufipogon · Sarocladium oryzae · Sheath rot

Wild rice (Oryza rufipogon Griff.) is the ancestor of Asian cultivated rice. In July 2020, sheath rot was observed on O. rufipogon (disease incidence of about 20\%) in an 8-ha field in Zhanjiang ( $\left.21^{\circ} 17^{\prime} 51^{\prime \prime N}, 110^{\circ} 18^{\prime} 16^{\prime \prime} \mathrm{E}\right)$, China. The symptoms were oblong dark brown lesions on sheaths with white mycelia. Three isolates (SoR-1, SoR-2, and SoR-3) were obtained from portions of surface sterilized diseased sheath incubated on potato dextrose agar (PDA) at $28^{\circ} \mathrm{C}$ for 5 days. Colonies were white. Conidiophores were branched. Phialides were cylindrical, 25-50×1.5-3.5 $\mu \mathrm{m}$ $(n=20)$. Conidia were hyaline, one-celled, and cylindrical, 5.0-12.5 $\mu \mathrm{m} \times 1.0-2.5 \mu \mathrm{m}(n=50)$. For molecular identification, colony PCR with MightyAmp DNA Polymerase (Lu et al. 2012) was used to amplify the internal transcribed spacer (ITS) and actin (ACT) loci of the isolates using primer pairs ITS1/ITS4 and ACT-512F/ACT-783R, respectively (Weir et al. 2012). In a phylogenetic analysis with concatenated ITS (accession Nos. MW478336 to MW478338) and ACT (MZ614957 to MZ614959) sequences, the three isolates clustered with $S$. oryzae CBS 180.74 type strain. Based on morphology characteristic and phylogenetic analysis, the isolates were identified as $S$. oryzae (Bills et al. 2004). Pathogenicity tests were performed in a greenhouse at $28^{\circ} \mathrm{C}$. Five plants (cv. Haihong- 12 at booting stage) for each strain were inoculated with $5 \mathrm{~mm}$-diameter mycelial plugs obtained from 5-day cultures. An equal number of plants was inoculated with PDA plugs as a control. The test was performed thrice. Symptoms observed 5 days after inoculation were similar to the sheath rot observed in the field. No disease was observed on controls. Reisolates from the inoculation group were identified as S. oryzae. To our knowledge, the study is the first report on S. oryzae causing sheath rot on $O$. rufipogon in China. Thus, vigilance is required for the utilization of $O$. rufipogon in breeding.

Supplementary information The online version contains supplementary material available at https://doi.org/10.1007/s42161-022-01090-6.

Funding This study was funded by Project of Enhancing School With Innovation of Guangdong Ocean University (GDOU2017052604).

\section{Declarations}

Human and animals rights This article does not contain any studies with human participants or animals performed by any of the authors.

Conflict of interest All authors declare that they have no conflict of interest.

\section{References}

Bills GF, Platas G, Gams W (2004) Conspecificity of the cerulenin and helvolic acid producing 'Cephalosporium caerulens', and the hypocrealean fungus Sarocladium oryzae. Mycol Res 108:1291-1300

Lu QF, Hu HQ, Mo JJ, Shu LZ (2012) Enhanced amplification of bacterial and fungal DNA using a new type of DNA polymerase. Austral Plant Pathol 41:661

Weir BS, Johnston PR, Damm U (2012) The Colletotrichum gloeosporioides species complex. Stud Mycol 73:115

Publisher's Note Springer Nature remains neutral with regard to jurisdictional claims in published maps and institutional affiliations.

Liu Yuelian

mushwoman@126.com

1 Guangdong Ocean University,

Zhanjiang, Guangdong 524088, China 